\title{
Lipid-based nanoparticles for photosensitive drug delivery systems
}

\author{
Gayong Shim ${ }^{1}\left[\right.$ (Sieon Jeong ${ }^{1} \cdot$ Jung Leem $\mathrm{Oh}^{1} \cdot$ Yeongseon Kang ${ }^{1}$
}

Received: 17 August 2021 / Accepted: 28 November 2021 / Published online: 6 January 2022

(c) The Author(s) under exclusive licence to The Korean Society of Pharmaceutical Sciences and Technology 2021

\begin{abstract}
Background Numerous drug delivery strategies have been studied, but many hurdles exist in drug delivery rates to the target site. Recently, researchers have attempted to remotely control the in vivo behavior of drugs with light to overcome the shortcomings of conventional drug delivery systems. Photodynamic and photothermal systems are representative strategies wherein a photosensitive material is activated in response to a specific wavelength of light.

Area covered Photosensitive materials generally exhibit poor solubility and low biocompatibility. Additionally, their low photostability negatively affects delivery performance. A formulation of lipid-based nanoparticles containing photosensitive substances can help achieve photosensitive drug delivery with improved biocompatibility. The lipid bilayer structure, which can be assembled and disassembled by modulating the surrounding conditions (temperature, $\mathrm{pH}$, etc.), can also be crucial for controlled release of drugs.

Expert opinion To the best of our knowledge, translation research on photoresponsive nanoparticles is scarce. However, as various drugs based on lipid nanoparticles have been clinically approved, the development potential of the lipid-based photoresponsive nanoparticles seems high. Thus, the identification of valid indications and development of optimum medical devices will increase the interest in photoresponsive material-based nanoparticles.
\end{abstract}

Keywords Lipid-based nanoparticles $\cdot$ Photosensitive materials $\cdot$ Triggered release $\cdot$ Chemo-phototherapy

\section{Introduction}

Drug delivery systems generally maximize the efficiency of drug delivery to a target site. Because of the enhanced permeability and retention effect, tumor targeting is possible even with simple nanoparticles (NPs) (Iyer et al. 2006; Shim et al. 2020a, b; Le et al. 2021). However, clinical studies have reported many controversial results and limitations associated with this effect. Therefore, ligand-modified NPs have been studied as an alternative for increasing the distribution to the target site (Le et al. 2019; Shim et al. 2020b; Yang et al. 2020). However, ligand-engineered NPs compensate for the shortcomings of conventional drugs only to some extent. Furthermore, these drug delivery systems do not impact the pharmacokinetic and biodistribution profiles of the drug, and many drugs were reportedly delivered to non-target sites or excreted from the body (Shim et al. 2017).

Gayong Shim

shim@ssu.ac.kr

1 School of Systems Biomedical Science, Soongsil University, Seoul 06978, Republic of Korea
Photoresponsive materials activated by light can help overcome the limitations of conventional drug delivery systems (Shim et al. 2017; Ko et al. 2018; Le et al. 2019; Wu et al. 2021). Photodynamic therapy (PDT) involves the activation of a drug by light of a specific wavelength. Several PDT agents have received FDA approval, and are currently being marketed (Mang 2004). Moreover, various substances that generate thermal energy by near-infrared (NIR) light are being studied as photothermal therapeutic agents (Ko et al. 2018; Le et al. 2019; Shim et al. 2020a, b; Yang et al. 2020; $\mathrm{Wu}$ et al. 2021). The most attractive feature of phototherapy is that the drug can be activated only in the target area. Many attempts have been made to apply photosensitizers in anticancer therapy as photosensitive mechanism induces apoptosis without causing drug resistance (Brackett and Gollnick 2011). However, most photosensitive materials have poor solubility and low biocompatibility. Porphyrin-based photosensitizers possess limited self-activation at non-target sites, and as a result, the treatment has to be performed in a dark room (Moriwaki et al. 2001). Additionally, safety concerns also limit the use of materials with photothermal effects (Miao, et al. 2013a, b; Miao, et al. 2013a, b). 
Lipid-based NPs can overcome the drawbacks of photosensitive materials owing to the following advantages (Fig. 1). First, lipid-based NPs used for drug delivery are known to be safe (Al-Jamal and Kostarelos 2011). Lipid NPs have been applied in a variety of platforms, from nanopharmaceuticals encapsulated with antibiotics or anticancer drugs to coronavirus disease vaccines (which have recently emerged as a hot topic); lipid NPs have thus been established as a good fit for nanomedicine (Pacheco et al. 2020; Schoenmaker et al. 2021). Second, lipid NPs are compatible with photosensitive materials. Lipid NPs are formed by selfassembly through hydrophobic interactions among lipids; this process can be easily controlled by controlling the ambient conditions. In other words, it is possible to change the structure of the lipid NPs by modulating the environment using a photosensitizer; this mechanism can be useful for controlling the release of drugs. It is also possible to introduce various moieties not only into the outer and inner spaces of the lipid NPs, but also into the space between the two layers of the lipid bilayer. Photosensitive substances and therapeutic agents with different physicochemical properties can be mounted in different spaces within the lipid NPs. Therefore, lipid NPs are highly suitable as drug delivery systems for combined phototherapy. We herein present a review of recent studies on photosensitive lipid NPs, focusing on the types of photosensitizers and the design of NPs. We also discuss the current status and challenges in translational research on these NP.

\section{Formulation of lipid NPs with photoresponsive materials}

\section{Porphyrin derivatives}

Studies on porphyrin-encapsulated lipid NPs are summarized in Table 1. A platinum-based anticancer drug (tetravalent platinum prodrug) and a photosensitizer (chlorin e6; Ce6) were co-incorporated into lipid NPs for anticancer photochemotherapy by controlling the redox balance (Yang et al. 2021). The platinum prodrug and $\mathrm{Ce} 6$ were inserted into the hydrophobic lipid bilayer to produce $\mathrm{Pt} / \mathrm{Ce} 6-\mathrm{NPs}$. While the mice in the control groups (saline, cisplatin, Ce6-NP + L, Pt-NP, and Pt/Ce6-NP groups) died within 15-30 days, the mice in the Pt/Ce6-NP + L group (irradiated with light) survived for more than 40 days. Compared to the other groups, the $\mathrm{Pt} / \mathrm{Ce} 6-\mathrm{NP}+\mathrm{L}$ group showed the lowest tumor growth rate and tumor volume (1000 $\mathrm{mm}^{3}$ at 25 days). Additionally, compared to that in the other groups, the expression of the M1-tumor associate macrophage marker (iNOS) was upregulated and that of the M2-tumor associate macrophage marker (CD206) was downregulated in the Pt/Ce6-NP + L group. The two drugs used in this study facilitated the induction of conditions for optimal functioning of both drugs. This helped in maximizing the anticancer effect. The selection of a combination of drugs that complement their respective anticancer

A

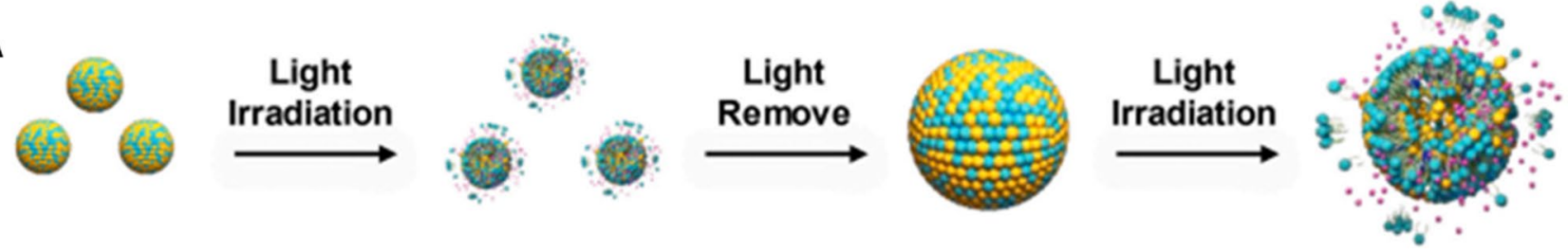

B
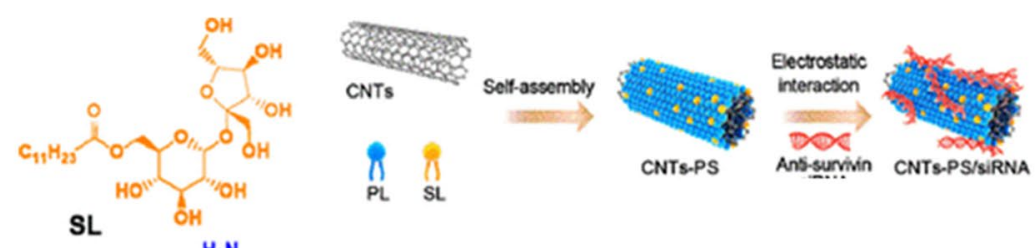

C
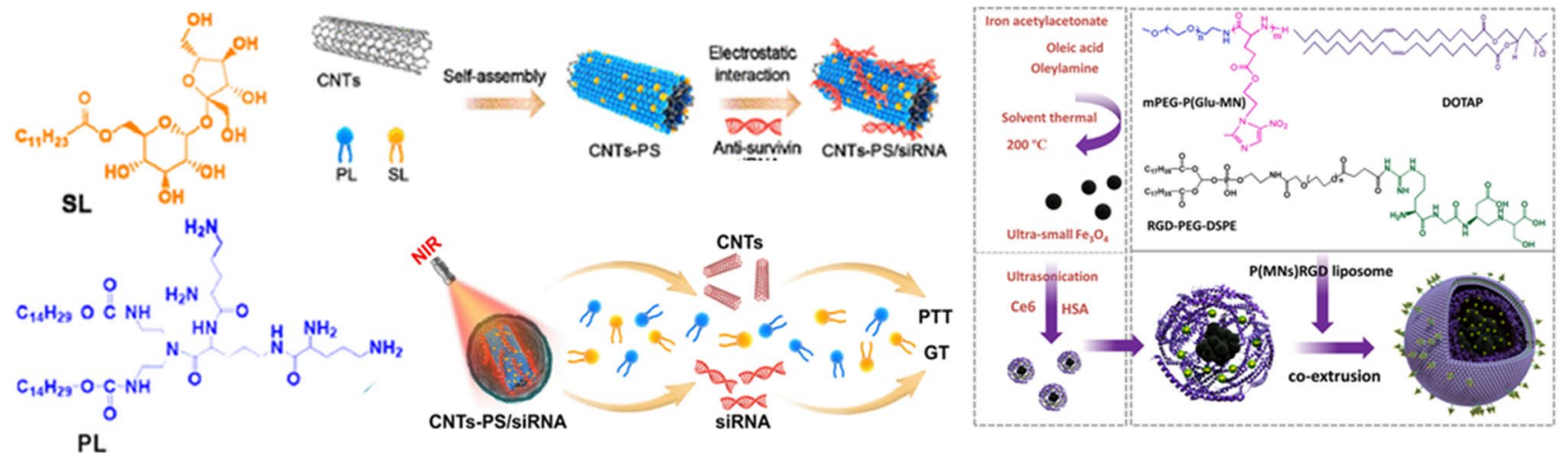

Fig. 1 Lipid-based photoresponsive drug delivery systems. A Platinum prodrug and Ce6-loaded lipid NP for anticancer photochemotherapy. Permission from Yang et al. (2021). B Lipid-coated carbon

nanotube for photothermal-gene therapy. Permission from Zhao et al. (2021). C Photosensitizer and iron oxide nanocluster-loaded lipid NP for photo/chemodynamic therapy. Permission from Tang et al. (2020) 
Table 1 Examples of porphyrin-encapsulated lipid nanoparticles

\begin{tabular}{|c|c|c|c|c|c|}
\hline $\begin{array}{l}\text { Photore- } \\
\text { sponsive } \\
\text { material }\end{array}$ & Nanocomposition & $\begin{array}{l}\text { Wave- } \\
\text { length } \\
(\mathrm{nm})\end{array}$ & Application & End point & References \\
\hline \multirow[t]{3}{*}{ Ce6 } & Cholesterol:DSPC:Egg yolk lecithin $=1: 1: 1(\mathrm{~m} / \mathrm{m})$ & 650 & $\begin{array}{l}\text { Chemo-PDT } \\
\text { On-demand drug release }\end{array}$ & In vivo study & Yang et al. (2021) \\
\hline & $\begin{array}{l}\text { Lecithin:Cholesterol:DSPE-mPEG2000:PEG- } \\
\text { NI:Ce6 } \\
=6: 4: 0.5: 0.5: 0.5(\mathrm{~m} / \mathrm{m})\end{array}$ & 670 & Chemo-PDT & In vivo study & Zhang et al. (2018) \\
\hline & $\begin{array}{l}\text { DSPE-PE-RGD:DOTAP:mPEG-poly(Glu-metro- } \\
\text { nidazole) } \\
=1: 2: 5\end{array}$ & $\begin{array}{l}660 \\
808\end{array}$ & Chemo-PDT/PTT & In vivo study & Tang et al. (2020) \\
\hline HMME & $\begin{array}{l}\text { DPPC: Cholesterol:DSPE-mPEG2000 = 50:5:5 } \\
(\mathrm{w} / \mathrm{w})\end{array}$ & 532 & $\begin{array}{l}\text { PDT } \\
\text { On-demand drug release }\end{array}$ & In vivo study & Shi et al. (2017) \\
\hline \multirow[t]{2}{*}{ mTHPC } & $\begin{array}{l}\text { DPPC:DSPC:DSPE-mPEG } 2000 \\
=85: 10: 5\end{array}$ & 650 & $\begin{array}{l}\text { Chemo-PDT } \\
\text { MHT }\end{array}$ & In vitro study & Shah et al. (2016) \\
\hline & $\begin{array}{l}\text { DPPC:Cholesterol =90:10 }(\mathrm{m} / \mathrm{m}) \\
\text { DPPC:DPPE-mPEG5000 =95:5 (m/m) } \\
\text { DPPC:Tetraether lipids }=90: 10(\mathrm{~m} / \mathrm{m})\end{array}$ & $\begin{array}{l}457 \\
652\end{array}$ & $\begin{array}{l}\text { PDT } \\
\text { On-demand drug release }\end{array}$ & In vivo study & Ali et al. (2020) \\
\hline \multirow[t]{3}{*}{ PoP } & $\begin{array}{l}\text { DSPC:DOTAP:Cholesterol:PoP } \\
=38: 20: 40: 2(\mathrm{~m} / \mathrm{m})\end{array}$ & 665 & Chemo-PDT & In vivo study & Luo et al. (2017) \\
\hline & DSPC or DSPC-mPEG:PoP = 1:19 $(\mathrm{m} / \mathrm{m})$ & 660 & PDT & In vivo study & Lovell et al. (2011) \\
\hline & $\begin{array}{l}\text { Sphingomyelin:PoP:Cholesterol } \\
=53: 2: 45(\mathrm{~m} / \mathrm{m})\end{array}$ & 665 & $\begin{array}{l}\text { Chemo-PDT } \\
\text { On-demand drug release }\end{array}$ & In vivo study & Carter et al. (2016) \\
\hline Verteporfin & $\begin{array}{l}\text { DOTAP:DOPC }=1: 1(\mathrm{~m} / \mathrm{m}) \\
\text { DOTAP:DOPC:Verteporfin } \\
=1: 0.94: 0.06(\mathrm{~m} / \mathrm{m})\end{array}$ & 365 & Photochemical internalization & In vitro study & Chen et al. (2017) \\
\hline
\end{tabular}

Ce6 Chlorin e6, HMME Hematoporphyrin monomethyl ether, $m T H P C$ 5,10,15,20-Tetrakis(3-hydroxyphenyl)chlorin, PoP Porphyrin-phospholipid, DSPC 1,2-distearoyl-sn-glycero-3-phosphocholine, DSPE-mPEG2O0O 1,2-distearoyl-ras-glycerol-3-phosphatidylethanolamine- $N$-polyethyleneglycol-2000, PEG-NI 2-nitroimidazole derivative-conjugated polyethylene glycol; 1,2-Distearoyl-sn-glycero-3-phosphoethanolaminepolyethylene glycol-RGD(ARG-GLY-ASP), DOTAP 1,2-dioleoyl-3-trimethylammonium-propane, DPPC dipalmitoylphosphatidylcholine, DPPE-mPEG5000 1,2-dipalmitoyl-sn-glycero-3-phosphoethanolamine-N-[methoxy(polyethylene glycol)-5000], DOPC 1,2-dioleoyl-sn-glycero3-phosphocholine, $P D T$ photodynamic therapy, $P T T$ photothermal therapy

effects is important for successful photochemotherapy, and lipid NPs are a suitable nanoplatform for this strategy.

A multifunctional hypoxia-responsive theranostic liposome was designed for PDT combined with chemotherapy (Zhang et al. 2018). Lipid NPs including lecithin, cholesterol, and nitroimidazole lipids were loaded with the photosensitizer $\mathrm{Ce} 6$ at the lipid bilayer and the hypoxia-activated prodrug tirapazamine in the aqueous phase. Upon red light irradiation, the dual drug-loaded lipid NPs induce severe hypoxia, leading to the disassembly of the lipid NPs and the activation of the prodrug tirapazamine. Nude mice bearing MCF7 tumors received the lipid NPs intravenously and 670$\mathrm{nm}$ laser irradiation treatment. Almost complete eradication of tumors was observed in the dual drug-loaded lipid NPtreated group.

Magnetite NPs of various sizes have been incorporated into photosensitive lipid NPs for multimodal therapies (Shah et al. 2016). The clinically approved hydrophobic drugs Foscan $(5,10,15,20$-tetrakis(3-hydroxyphenyl; mTHPC) and doxorubicin (DOX) were co-loaded into the lipid bilayer of lipid NPs. Magnetic NPs of three different sizes (10, 22, and 30-nm) were encapsulated in the aqueous phase of lipid NPs (40, 70, and 110-nm, respectively). Among these, the lipid NPs with intermediate size encapsulated with 22-nm magnetic NPs were found to be the most suitable candidates for evaluating the magnetic properties and colloidal stability. The effects of PDT, magnetic hyperthermia therapy, and chemotherapy, either alone or in combination, were investigated in HeLa cervical cancer cells. Combination therapy including all the three treatment modalities induced apoptosis in more than $90 \%$ of the cancer cells. The anticancer effect of the combination therapy was much stronger than that of monotherapy with each of the three modalities.

The optimal formulation of liposomal temoporfin for PDT was evaluated using ovarian cancer cells (Ali et al. 2020). The drug mTHPC was incorporated into 1,2-dipalmitoyl-sn-glycero-3-phosphatidylcholine-based lipid NPs and tested with two different wavelengths of light. To stabilize the membrane structure, tetraether lipids, which are abundant in the plasma membrane of archaea, were used. A series of liposome candidates showed an mTHPC encapsulation rate of $\geq 75 \%$. The mTHPC was activated 
in response to two wavelengths of light. For the liposomal temporfin prepared in this study, the PDT effect was higher at $652-\mathrm{nm}$ than at $457-\mathrm{nm}$. A composition containing a small fraction of polyethylene glycol lipids has been reported to have the highest PDT effect (Ali et al. 2020). The photo-thrombic ability of mTHPC lipid NPs was confirmed by complete local vascular destruction at $24 \mathrm{~h}$ after treatment.

Cationic liposomes containing porphyrin-derived lipids have been evaluated for tumor blood vessel-targeting activity (Luo et al. 2017). Lovell et al. developed lipid NPs composed of porphyrin-phospholipid (PoP) conjugates that can be permeabilized by porphyrin-specific light (Lovell et al. 2011). In this study, the cationic lipid 1,2-dioleoyl3-trimethylammonium-propane (DOTAP) and DOX were introduced into lipid NPs for tumor vessel targeting and anticancer therapy, respectively. Although the mechanism by which cationic lipid NPs target tumor endothelial cells has not been elucidated, it may involve interactions with anionic macromolecules that show increased expression during angiogenesis. Light irradiation can release DOX from the DOTAP/PoP lipid NPs through PoP-dependent unsaturated lipid photo-oxidation. In this study, the DOX DOTAP/PoP NPs (+ laser) group showed the highest reduction in tumor volume, at $91 \%$. In addition, the empty (free DOX) DOTAP NPs (+ laser) group unexpectedly had an effect similar to that observed in the DOX DOTAP/PoP NPs (+laser) group. Tumor growth inhibition was not observed in any of the other groups. There was no significant difference in tumor volume between the DOX DOTAP/PoP NPs (-laser) group and the saline group. In other words, the anti-tumor activity might be attributed to phototherapy rather than to chemotherapy. When the light intensity was reduced by $80 \%$ and the drug dose was increased by 1.4 times, tumor growth in the empty DOTAP NPs (+ laser) group and the DOX DOTAP/PoP NPs (+laser) group was suppressed by $57 \%$ and $85 \%$, respectively, compared to that in the saline group. In clinical practice, depending on the tumor size, there may be areas where the light intensity is lower than expected. Therefore, these results are meaningful in terms of photochemotherapy.

The same group studied the composition of the PoPlipid NPs to overcome the problem of PoP interference with respect to DOX loading (Carter et al. 2016). The anticancer drug was changed to irinotecan, which has good compatibility with PoP, and the composition of the liposome was optimized so that sphingomyelin and cholesterol were the main components. Because irinotecan is unstable in liposomal formulations, polyethylene glycol was excluded for a stable loading of the drug. Compared to polyethylene glycolmodified NPs, the serum stability of polyethylene glycolfree formulation was sub-optimal, but this system eradicated tumors in MIA Paca-2 subcutaneous tumor xenografts.
Enhanced photochemical internalization of nucleic acid drugs has been studied using photoresponsive lipid NPs (Chen et al. 2017). A mixture of DOTAP, 1,2-dioleoyl-snglycero-3-phosphocholine, and photosensitizer verteporfin (VP) was evaporated to prepare VP-loaded lipid NPs. Then, an antisense oligonucleotide (asODN) targeting the PAC1R (pituitary adenylyl cyclase-activating polypeptide receptor 1) gene was incorporated into the aqueous phase of the lipid NPs by thin-film hydration. AsODN was taken up by PC12 cells through the lipid NPs, and remained in the endosome for $4 \mathrm{~h}$. However, when the cells were treated with $\mathrm{VP} /$ asODN-LP and irradiated with light $3 \mathrm{~h}$ later, it was observed that the asODN was released from the endosomes. This result indicates that when light irradiation causes VPmediated ROS generation in lipid NPs, photochemical internalization of asODN is induced, leading to asODNmediated gene silencing. There was a $74 \%$ reduction in the fluorescence intensity pertaining to the fluorescence-labeled PAC1R protein after treatment with VP/asODN-LP (+light).

\section{Fluorescent imaging dyes}

Studies on fluorescence dye-encapsulated lipid NPs are summarized in Table 2. Phototherapy-mediated phase transition of a lipid bilayer was studied using gold nanorod or indocyanine green-encapsulated liposomes (Viitala et al. 2016). When exposed to 808-nm laser, both photothermal agents were observed to convert light into heat. The heat increase caused phase transitions in the lipid bilayer, resulting in the release of the model drug calcein. Liposomal indocyanine green and gadolinium $(\mathrm{Gd})$ were formulated for multimodal imaging and combination therapy (Dai et al. 2019). The lipid NPs were decorated with lipophilic Gd and folic acidderived lipids for magnetic resonance imaging and tumor targeting, respectively. In the aqueous core of liposomes, the IR dye indocyanine green and DOX were co-encapsulated to form multimodal NPs. This nanoplatform was able to release the chemotherapeutic drug upon NIR irradiation and exert photothermal and PDT effects. Multimodal NPs are multifunctional imaging agents and are also used for fluorescence, magnetic resonance, and photoacoustic imaging. Enhanced tumor accumulation of the nanoparticles was observed in HeLa-xenografted mice after systemic administration. Upon NIR irradiation, the temperature of the tumor increased to $61{ }^{\circ} \mathrm{C}$ in the ID@TSL-Gd NP group, and tumor growth was arrested to a large extent.

Selective activation of autophagy inducers has been demonstrated by an IR dye-loaded liposomal formulation (Appidi et al. 2020). The chlorophyll-rich fraction of Anthocephalus cadamba (CfAc) was used as a bioactive substance; this substance has been reported to induce ROS. CfAc and the photosensitizer IR780 were loaded into liposomes by the thin-film hydration method to produce NPs 
Table 2 Examples of fluorescence imaging dye-encapsulated lipid nanoparticles

\begin{tabular}{|c|c|c|c|c|c|}
\hline Photoresponsive material & Nanocomposition & $\begin{array}{l}\text { Wave- } \\
\text { length } \\
(\mathrm{nm})\end{array}$ & Application & Endpoint & References \\
\hline \multirow[t]{2}{*}{ ICG } & DPPC & 808 & On-demand drug release & Proof-of-concept & Viitala et al. (2016) \\
\hline & $\begin{array}{l}\text { Lauric acid:Stearic acid=4:1 } \\
(\mathrm{w} / \mathrm{w}) \\
\text { Gd - DTPA - SA:DSPE- } \\
\text { mPEG2000-folic } \\
\text { acid:Lecithin }=1: 1: 3(\mathrm{w} / \mathrm{w})\end{array}$ & 808 & $\begin{array}{l}\mathrm{PTT} / \mathrm{PDT} / \\
\text { On-demand drug release }\end{array}$ & In vivo study & Dai et al. (2019) \\
\hline IR780 & $\begin{array}{l}\text { DSPC:CfAc }=10: 0.75(\mathrm{w} / \mathrm{w}) \\
\text { DSPC:IR780 }=10: 0.25(\mathrm{w} / \mathrm{w})\end{array}$ & 808 & PDT & In vivo study & Appidi et al. (2020) \\
\hline Methylene blue & $\begin{array}{l}\text { DPPC:Cholesterol:DOTAC } \\
=1: 0.5:(\mathrm{w} / \mathrm{w})\end{array}$ & 633 & PDT & In vitro study & Boccalini et al. (2017) \\
\hline
\end{tabular}

ICG indocyanine green, DPPC 1,2-Dipalmitoyl-sn-glycero-3-phospho-choline, Gd-DTPA-SA gadolinium-diethylenetriaminepentaacetic dihydride-stearylamine, DSPC 1,2-distearoyl-sn-glycero-3-phosphocholine, DOTAC dimethyldioctadecylammonium chloride, PDT photodynamic therapy, $P T T$ photothermal therapy

containing CfAc (CfAc NPs) and NPs containing IR780 (IR NPs). Without NIR irradiation, the MCF7 cells treated with CfAc NPs, IR NPs, or CIR NPs (NPs containing both CfAc and IR780) showed no changes in ROS and autophagy levels. In contrast, when the NP-treated MCF cells were irradiated with NIR light, the CIR NP group showed the highest levels of ROS and consequently exhibited the induction of autophagy. The in vivo administration of IR or CIR NPs $(+\mathrm{NIR})$ reduced $4 \mathrm{~T} 1$ tumor volume by more than $88 \%$ and $95 \%$, respectively, compared to the saline group. These results suggest that photoresponsive liposomes facilitate the spatiotemporal control of ROS generation.

The antibacterial activity of methylene blue (MB) was investigated using a liposomal formulation (Boccalini et al. 2017). MB, which is both a fluorescent dye and an approved medication (Provayblue $\AA$ ) for methemoglobinemia, can generate ROS under red light irradiation. MBloaded lipid NPs were prepared with the cationic lipid dimethyldioctadecylammonium chloride, which enabled the NPs to interact with negatively charged bacterial membranes. Liposomal MB (+ light) showed enhanced bacterial toxicity and biofilm penetrating activity when compared with free MB. Moreover, liposomal MB is reported to have an anti-inflammatory effect leading to the inactivation of lipopolysaccharides.

\section{Metal-based materials}

Examples of metal/lipid NPs are summarized in Table 3. Liposomes coated with gold NPs (GNPs) were utilized to induce hyperthermia upon NIR light irradiation for drug release and chemo-phototherapy (Luo et al. 2016). Lipid NPs loaded with the anticancer agent oleanolic acid (OA) were coated with chitosan to enhance stability and to promote cancer cell uptake. Subsequently, gold nanoshellcoated NPs were prepared by the seed growth method (Jana

Table 3 Examples of metal-encapsulated lipid nanoparticles

\begin{tabular}{|c|c|c|c|c|c|}
\hline Photoresponsive material & Nanocomposition & $\begin{array}{l}\text { Wave- } \\
\text { length } \\
(\mathrm{nm})\end{array}$ & Application & Endpoint & References \\
\hline \multirow[t]{3}{*}{ Gold nanoparticle } & $\begin{array}{l}\text { Soya lecithin:cholesterol:oleanolic } \\
\text { acid }=50: 6: 5(\mathrm{w} / \mathrm{w})\end{array}$ & 808 & $\begin{array}{l}\text { PTT } \\
\text { On-demand drug release }\end{array}$ & In vivo efficacy & Luo et al. (2016) \\
\hline & $\begin{array}{l}\text { DPPC:DSPE-mPEG2000 = 30:6 } \\
(\mathrm{w} / \mathrm{w})\end{array}$ & 800 & $\begin{array}{l}\text { Chemo-PTT } \\
\text { On-demand drug release }\end{array}$ & In vivo study & Li et al. (2018) \\
\hline & $\begin{array}{l}\text { Phospholipon 90G:Cholesterol=7:0, } \\
\text { 7:1, 7:2, 7:7, 7:14, and 7:28 }\end{array}$ & 690 & On-demand drug release & In vitro study & Wiraja et al. (2016) \\
\hline Iridium nanocrystals & $\begin{array}{l}\text { DPPC:Cholesterol:DSPE- } \\
\text { mPEG5000 = 6:4:0.5 }\end{array}$ & 785 & $\mathrm{PTT} / \mathrm{RT}$ & In vivo study & Feng et al. (2018) \\
\hline $\mathrm{Au}-\mathrm{Pd}$ bimetallic nanoflowers & $\begin{array}{l}\text { Soya lecithin:Cholesterol:Betulinic } \\
\text { acid }=50: 6.2: 5(\mathrm{w} / \mathrm{w})\end{array}$ & 808 & $\begin{array}{l}\text { PTT } \\
\text { On-demand drug release }\end{array}$ & In vivo study & Liu et al. (2018) \\
\hline
\end{tabular}

$D P P C$ 1,2-dipalmitoyl-sn-glycero-3-phospho-choline, DSPE-mPEG5000 1,2-distearoyl-ras-glycerol-3-phosphatidylethanolamine- $N$-polyethyleneglycol-5000, $P T T$ photothermal therapy $R T$ radiotherapy 
et al. 2001). GNP-triggered phase transition of lipid NPs was observed to release OA (Luo et al. 2016). In vivo efficacy data showed that the saline and laser-alone groups did not show tumor growth inhibition, and the free OA group showed tumor growth inhibition by approximately $50 \%$ compared to the saline group. Similarly, compared to the free OA group, the chitosan-coated OA NP group and the gold/ chitosan-coated OA NP (-NIR) group showed tumor growth inhibition, and the group with the highest level of tumor suppression was the gold/chitosan-coated OA NP (+ NIR) group (79.65\% inhibition rate). Upon irradiation of the lipid NPs with NIR light, the surrounding GNPs absorb light and generate heat, promoting the death of cancer cells and enabling the rapid release of OA.

Hollow GNPs (HGNPs) or GNPs were used as nanoswitches to trigger drug release from thermosensitive lipid NPs (Li et al. 2018). HGNPs are hollow spheres and have greater photothermal conductivity than GNPs due to their NIR surface plasmon tunability. Thermosensitive lipid NPs were formulated using 1,2-dipalmitoyl-sn-glycero-3-phosphocholine; this lipid was selected due to its relatively low phase transition temperature $\left(41^{\circ} \mathrm{C}\right)$. The lipid film was hydrated with the HGNP or GNP solution to produce HGNP-NPs or GNP-NPs, respectively. DOX was then incorporated into the liposomes by the remote loading method. DOX was released from the lipid NPs upon NIR irradiation which caused hyperthermia-mediated destruction of the lipid NPs. HGNP has a much higher light-to-heat conversion efficiency than GNP-in fact, GNP requires five times the amount of light required by HGNP to reach the same temperature. Poly(vinylpyrrolidone), which is used as a stabilizing agent in HGNP synthesis, was reported to contribute to the red-shifted surface plasmon resonance absorption peak at $800-\mathrm{nm}$. Treatment with DOX/HGNPs (+ NIR) showed a remarkable reduction in tumor volume that was 2.77 - or 2.12-fold lower than that due to treatment with free DOX or HGNP-TL (+ NIR), respectively.

Liposomal iridium nanocrystals (IrNCs) have been applied to overcome hypoxic conditions in solid tumors, resulting in improved efficacy of radiotherapy (Feng et al.
2018). Liposomal IrNCs exert catalytic activity to degrade $\mathrm{H}_{2} \mathrm{O}_{2}$ to $\mathrm{O}_{2}$ under NIR treatment, leading to increased tumor oxygenation; this increased oxygenation was reported to sensitize the tumor tissue to radiotherapy. Liposomal IrNCs were formulated for prolonged blood circulation and tumor retention. An intravenous injection of IrNC in 4T1 tumorbearing mice showed saturated tumor accumulation of the IrNCs at $8 \mathrm{~h}$ after injection. Moreover, liposomal IrNCs caused a significant decrease in hypoxia levels in tumors after NIR irradiation. Mice treated with liposomal IrNCs and exposed to NIR irradiation and X-ray radiation (6 Gy) showed significantly suppressed tumor growth compared to the mice in the groups that received either phototherapy or radiotherapy alone.

Poly-branched Au-Pd bimetallic-nanoflower-coated betulinic acid lipid NPs (BA-Lips@Pd@Au NFs) showed great potential when applied in photothermal therapy (PTT) combined with chemotherapy (Liu et al. 2018). The BA-Lips@ Pd@Au NFs were composed of soya lecithin, cholesterol, and betulinic acid, and coated with Pd seeds and Au growth solution in a layer-by-layer manner. The BA-Lips@Pd@ $\mathrm{Au}$ NFs can generate heat upon 808-nm laser irradiation; this leads to phase transition and triggers drug release. BALips@Pd@Au NFs were intratumorally injected into mice bearing U14 cells under 808-nm irradiation. These NPs caused tumor cell ablation due to local hyperthermia, and thus exhibited antitumor activity.

\section{Photoswitchable chemicals}

Studies on photoswitchable chemical-encapsulated lipid NPs are summarized in Table 4. A photoswitchable system was formulated by introducing an azobenzene moiety into lipid NPs (Liu and An, 2019). Phosphatidyl choline-based lipid NPs were used for encapsulating curcumin and 4-butylazobenzene-4-hexyloxy-trimethyl-ammoniumtrifluoroacetate (BHA), which can be isomerized upon ultraviolet (UV) irradiation. BHA inserted into the BHA-curcumin-NP isomerizes in response to light, and curcumin is released when the lipid bilayer structure of the liposome is breached.

Table 4 Examples of photoswitchable chemical-encapsulated lipid nanoparticles

\begin{tabular}{llllll}
\hline Photoresponsive material & Nanocomposition & $\begin{array}{l}\text { Wave- } \\
\text { length } \\
(\mathrm{nm})\end{array}$ & Application & Endpoint & References \\
\hline BHA & Egg phosphatidyl choline & 348 & On-demand drug release & Proof-of-concept & Liu et al. (2019) \\
Molecular motor & DOPC & 365 & On-demand drug release & Proof-of-concept & Ribovski et al. (2020) \\
Azobenzene moiety & POPC & 365 & On-demand drug release & Proof-of-concept & Hernández-Ainsa et al. (2016) \\
& & 420 & & & \\
\hline
\end{tabular}

BHA 4-butylazobenzene-4-hexyloxy-trimethyl-ammoniumtrifluoro-acetate, molecular motor 5,5'-(9-(2-methyl-2,3-dihydro-1H-cyclopenta[a] naphthalen-1-ylidene)-9H-fluorene-3,6-diyl)diisophthalic acid, DOPC 1,2-dioleoyl-sn-glycero-3-phosphocholine, POPC 2-oleoyl-1-palmitoylsn-glycero-3-phosphocholine 
Approximately $90 \%$ of the curcumin in the BHA- curcuminNP was observed to be released in $6 \mathrm{~h}$ under UV irradiation. Additionally, UV and visible light irradiation were able to alternatively control drug release.

UV-induced rotation of molecular motor liposomes can facilitate the release of cargo molecules (Ribovski et al. 2020). The tetrapodal molecular motor (5,5'-(9-(2-methyl2,3-dihydro-1H-cyclopenta[a]naphthalen-1-ylidene)-9H-fluorene-3,6-diyl)diisophthalic acid) can be loaded into the hydrophobic regions of the lipid NPs and unidirectionally rotated using UV light. Photoisomerization of molecular motors in liposomes can lead to disassembly of the nanostructures, resulting in the release of the cargo drug.

DNA oligonucleotide-attached lipid NPs can be assembled or disassembled by various stimuli including UV light (Hernández-Ainsa et al. 2016). Trans-azobenzene-oligonucleotide (AZO-ON) and cis-AZO-ON-modified lipid NPs showed differences in the degree of lipid NP assembly. While it was easy to assemble lipid NPs with trans-AZO$\mathrm{ON}$, it was more difficult to assemble lipid NPs with cisAZO-ON. When irradiated with light at a wavelength of 365-nm, trans-AZO-ON gets converted to cis-AZO-ON; irradiation with light at a wavelength of 420-nm causes the conversion of cis-AZO-ON to trans-AZO-ON. Thus, the assembly and disassembly of lipid NPs can be controlled by irradiating with the light of different wavelengths. This concept can be applied to regulate drug release and control the behavior of lipid NPs.

\section{Carbon-based materials}

Studies on carbon-modified lipid NPs are summarized in Table 5. Lipid-coated carbon nanotubes were utilized in conjunction with small interfering RNA for photothermal gene therapy (Zhao et al. 2021). Carbon nanotubes were coated with peptide lipids and sucrose laurate, and this complex exhibited a phase transition temperature of $42{ }^{\circ} \mathrm{C}$. Due to the positive charge of the peptide lipid, small interfering RNA can be loaded onto the carbon nanotube surface. Irradiation with NIR light leads to an increase in temperature, which releases the lipid layer from carbon nanotube; this consequently results in the release of the small interfering RNA into the cytoplasm.

Liposomal DOX was coated with graphene oxide for chemo-PTT (Hashemi et al. 2018). Graphene-coated liposomes were prepared using the layer-by-layer method. First, DOX was incorporated into the cationic lipid NPs through remote loading. Then, the surface of the cationic liposome was coated with graphene oxide by electrostatic attraction. Finally, the liposome was coated with the graphene oxide modified with poly-L-lysine (a cationic polymer) to obtain graphene-coated liposomes. The outer layer of the graphene-coated liposomes contributes to the intracellular delivery of the drug because of its positive charge. Because the layer-by-layer method uses electrostatic attraction and can easily cause precipitation, it is important to optimize the concentration of each component to formulate stable NPs. Further studies on in vivo applications of these liposomes are required.

\section{Polymers}

Studies on polymer-modified lipid NPs are summarized in Table 5. A malachite green moiety was adopted as a photoresponsive material to trigger the release of drugs from lipid NPs (Uda et al. 2016). Lecithin-based lipid NPs were loaded with a fluorescent dye. The lipid NPs were formulated with egg yolk lecithin and chloroform, and the fluorescent dye was encapsulated at the aqueous core phase. Under UV light, the malachite moiety photoisomerizes, and the positively charged moiety binds to the lipid NPs through electrostatic interactions. When the malachite moiety binds to the lipid

Table 5 Examples of carbon or polymer-modified lipid nanoparticles

\begin{tabular}{|c|c|c|c|c|c|}
\hline Photoresponsive material & Nanocomposition & $\begin{array}{l}\text { Wave- } \\
\text { length } \\
(\mathrm{nm})\end{array}$ & Application & Endpoint & References \\
\hline $\begin{array}{l}\text { Single-walled carbon nano- } \\
\text { tubes and multi-walled } \\
\text { carbon nanotubes }\end{array}$ & $\begin{array}{l}\text { Peptide Lipid:Sucrose laurate }=1: 1 \\
(\mathrm{w} / \mathrm{w})\end{array}$ & 808 & $\begin{array}{l}\text { PTT } \\
\text { On-demand drug release }\end{array}$ & In vivo study & Zhao et al. (2021) \\
\hline $\begin{array}{l}\text { Graphene oxide, graphene } \\
\text { oxide-conjugated poly(L- } \\
\text { lysine) }\end{array}$ & $\begin{array}{l}\text { DPPC:Brijß S20: } \\
\text { DOTAP:Cholesterol = 66:4:20:10 } \\
\quad(\mathrm{m} / \mathrm{m})\end{array}$ & 808 & $\begin{array}{l}\text { Chemo-PTT } \\
\text { On-demand drug release }\end{array}$ & In vitro study & Hashemi et al. (2018) \\
\hline Malachite green copolymer & Egg yolk lecithin & 625 & On-demand drug release & Proof-of-concept & Uda et al. (2016) \\
\hline Polydopamine & $\begin{array}{l}\text { DPPC:DPPG:DSPE-mal- } \\
\text { PEG2000 = 7:3:0.1 (m/m) }\end{array}$ & 808 & PTT & In vivo study & Yang et al. (2020) \\
\hline
\end{tabular}

GO graphene oxide, GO-PLL graphene oxide-conjugated poly(L-lysine); Brijß S20, polyoxyethylene (20) stearyl ether, DOTAP 1,2-dioleoyl3-trimethylammonium-propane, $D P P C$ 1,2-dipalmitoyl-sn-glycero-3-phospho-choline, $D P P G$ 1,2-dipalmitoyl-sn-glycero-3-phosphorylglycerol, DSPE-malPEG2000 1,2-distearoyl-sn-glycero-3-phosphoethanolamine-N-[maleimide(polyethylene glycol)-2000], PTT photothermal therapy 
NPs, it releases the encapsulated fluorescent dye due to membrane destabilization.

Lipid/polydopamine hybrid NPs have been formulated for tumor-targeted PTT (Yang et al. 2020). Anionic lipid NPs, including maleimide-conjugated lipids, were co-extruded with polydopamine to produce maleimide-modified lipid/ polydopamine hybrid NPs. Anti-claudin 3 antibody was added to the NPs through a maleimide-thiol reaction. Antibody-modified NPs bind strongly to tumor cells in a claudin 3-specific manner. The increased accumulation of NPs in the tumor results in a potent photothermal ablation of the tumor.

\section{Translational research and perspectives}

PDT is the mainstay of phototherapy, and several drugs have been approved by the Food and Drug Administration (Allison et al. 2004) in this regard (Table 6). Most of these drugs have been approved for use in dermal applications. When free drugs are administered, it is impossible to target a specific organ or lesion, and there is a high possibility that they will eventually be activated in a non-target organ. In addition, because the depth of light irradiation is shallow, the range of indications is limited if the light is applied to the body externally (Arslan et al. 2018). One solution is the development of medical devices that facilitate the endoscopic application of light. In the case of Photofrin ${ }^{\circledR}$, an optiguide fiber optic diffuser was developed for irradiating the lungs using endoscopically applied light (Harewood et al. 2005).

A nanoformulation of photosensitizers is a promising solution to control the systemic distribution and activity of photosensitizers. Despite the various research results described above, a photosensitizer drug formulated with NPs has not yet been developed. Currently, a phase $1 / 2$ clinical trial (NCT02367547) is underway to study the application of PDT against cancer using a nanoscale lipid vesicle gel formulation (Salmivuori et al. 2020). In addition, PTT therapy using silica-GNPs has been evaluated in a human study (NCT01270139). PTT using implanted NPs has been applied for the treatment of atherosclerosis.
Significant lesion reduction was observed after treatment, and the side effects were less severe than those with stenting (Kharlamov et al. 2015, 2017).

Among the liposomal drugs currently being tested in clinical trials, ThermoDox ${ }^{\circledR}$ is noteworthy (Swenson et al. 2015; Lyon et al. 2017). ThermoDox ${ }^{\circledR}$ is a system that releases DOX sealed in lysolipid thermally sensitive liposomes at temperatures greater than $40{ }^{\circ} \mathrm{C}$. Thus, it is possible to achieve a local DOX concentration in the lesion that is 25-fold higher than that achieved by conventional systemic administration (Bulbake et al. 2017). A phase 3 clinical trial in primary liver cancer patients is underway to evaluate the effectiveness of ThermoDox $® / O P T I M A$. This system is used in combination with thermal treatments such as ThermoDox ${ }^{\circledR}$ and radiofrequency thermal ablation, microwave hyperthermia, or high-intensity focused ultrasound (Tagami and Ozeki 2017). ThermoDox ${ }^{\circledR}$ alone is being evaluated preclinically for efficacy against bladder cancer. If an external stimulus that generates heat is replaced with a light source, then the expandability of this nanoplatform will be high.

\section{Conclusion}

Although there have been many interesting studies, photoresponsive material-based NPs that can be applied clinically are yet to be developed. However, several lipid NPs have already been clinically approved. The fact that there are many approved lipid nanoparticles implies that the lipid NPs are relatively safe, and that experience has been accumulated with regard to the manufacturing process. For the development of photoresponsive material-based NPs, an appropriate lipid formulation should be developed to increase the biocompatibility of the photosensitive materials. In addition, medical devices capable of applying light locally to the lesion should be developed. Although several challenges remain, photosensitive lipid nanoparticles show potential for application as a non-invasive treatment technology.

Table 6 FDA-approved photodynamic therapy agents

\begin{tabular}{|c|c|c|c|c|}
\hline PDT agent & Photosensitizer & Approval year & Wavelength (nm) & Indication \\
\hline Levulan® & PpIX & 1999 & $\begin{array}{l}400-450 \\
(\text { Blu-Uß) } \\
600-650\left(\text { Aktilite }^{\mathrm{TM}}\right)\end{array}$ & $\begin{array}{l}\text { Actinic keratoses on the face and scalp } \\
\text { Squamous precancer of the skin }\end{array}$ \\
\hline Photofrin ${ }^{\circledR}$ & Porfimer sodium & 2003 & 630 & Esophageal cancer \\
\hline Metvix ${ }^{\circledR}$ & PpIX & 2004 & $\begin{array}{l}630 \\
570-670\end{array}$ & $\begin{array}{l}\text { Actinic keratoses on the face and scalp } \\
\text { Basal cell carcinoma Unsuitable for } \\
\text { other available therapies }\end{array}$ \\
\hline
\end{tabular}

PpIX protoporphyrin IX, FDA Food and Drug Administration, $P D T$ photodynamic therapy 
Acknowledgements This study was supported by the Basic Science Research Program through the National Research Foundation of Korea (NRF) funded by the Ministry of Education (NRF2020R1I1A1A01070084), the Korea Medical Device Development Fund grant funded by the Korean government (the Ministry of Science and ICT; Ministry of Trade, Industry and Energy; Ministry of Health \& Welfare; and Ministry of Food and Drug Safety) (NTIS Number: 9991007273, KMDF_PR_20200901_0106), and the Korean Health Technology R\&D Project (No. HI19C0664).

\section{Declarations}

Conflict of interest All authors (G. Shim, S. Jeong, J.L. Oh, and Y. Kang) declare that they have no conflict of interest.

Statement of human and animal rights This article does not any studies with human and animal subjects performed by any of the authors.

\section{References}

Ali S, Amin MU, Ali MY, Tariq I, Pinnapireddy SR et al (2020) Wavelength dependent photo-cytotoxicity to ovarian carcinoma cells using temoporfin loaded tetraether liposomes as efficient drug delivery system. Eur J Pharm Biopharm 150:50-65

Al-Jamal WT, Kostarelos K (2011) Liposomes: From a clinically established drug delivery system to a nanoparticle platform for theranostic nanomedicine. Acc Chem Res 44:1094-1104

Allison RR, Mota HC, Sibata CH (2004) Clinical PD/PDT in North America: a historical review. Photodiagn Photodyn Ther $1: 263-277$

Appidi T, Pemmaraju DB, Khan RA, Alvi SB, Srivastava R et al (2020) Light-triggered selective ROS-dependent autophagy by bioactive nanoliposomes for efficient cancer theranostics. Nanoscale 12:2028-2039

Arslan H, Doluğan YB, Ay AN (2018) Measurement of the penetration depth in biological tissue for different optical powers. Sakarya Üniversitesi Fen Bilimleri Enstitüsü Dergisi 22:1095-1100

Boccalini G, Conti L, Montis C, Bani D, Bencini A et al (2017) Methylene blue-containing liposomes as new photodynamic anti-bacterial agents. J Mater Chem B 5:2788-2797

Brackett CM, Gollnick SO (2011) Photodynamic therapy enhancement of anti-tumor immunity. Photochem Photobiol Sci 10:649-652

Bulbake U, Doppalapudi S, Kommineni N, Khan W (2017) Liposomal formulations in clinical use: An updated review. Pharmaceutics 9:12

Carter KA, Luo D, Razi A, Geng J, Shao S et al (2016) Sphingomyelin liposomes containing porphyrin-phospholipid for irinotecan chemophototherapy. Theranostics 6:2329-2336

Chen W, Deng W, Goldys EM (2017) Light-triggerable liposomes for enhanced endolysosomal escape and gene silencing in PC12 cells. Mol Ther Nucleic Acids 7:366-377

Dai Y, Su J, Wu K, Ma W, Wang B et al (2019) Multifunctional thermosensitive liposomes based on natural phase-change material: Near-infrared light-triggered drug release and multimodal imaging-guided cancer combination therapy. ACS Appl Mater Interfaces 11:10540-10553

Feng L, Dong Z, Liang C, Chen M, Tao D et al (2018) Iridium nanocrystals encapsulated liposomes as near-infrared light controllable nanozymes for enhanced cancer radiotherapy. Biomaterials 181:81-91

Harewood GC, Baron TH, Rumalla A, Wang KK, Gores GJ et al (2005) Pilot study to assess patient outcomes following endoscopic application of photodynamic therapy for advanced cholangiocarcinoma. J Gastroenterol Hepatol 20:415-420

Hashemi M, Omidi M, Muralidharan B, Tayebi L, Herpin MJ et al (2018) Layer-by-layer assembly of graphene oxide on thermosensitive liposomes for photo-chemotherapy. Acta Biomater 65:376-392

Hernández-Ainsa S, Ricci M, Hilton L, Aviñó A, Eritja R et al (2016) Controlling the reversible assembly of liposomes through a multistimuli responsive anchored DNA. Nano Lett 16:4462-4466

Iyer AK, Khaled G, Fang J, Maeda H (2006) Exploiting the enhanced permeability and retention effect for tumor targeting. Drug Discov Today 11:812-818

Jana NR, Gearheart L, Murphy CJ (2001) Wet chemical synthesis of high aspect ratio cylindrical gold nanorods. J Phys Chem B 105:4065-4067

Kharlamov AN, Tyurnina AE, Veselova VS, Kovtun OP, Shur VY et al (2015) Silica-gold nanoparticles for atheroprotective management of plaques: results of the NANOM-FIM trial. Nanoscale 7:8003-8015

Kharlamov AN, Feinstein JA, Cramer JA, Boothroyd JA, Shishkina EV et al (2017) Plasmonic photothermal therapy of atherosclerosis with nanoparticles: long-term outcomes and safety in NANOM-FIM trial. Future Cardiol 13:345-363

Ko S, Shim G, Kim J, Oh Y-K (2018) Chemokine-mimetic plerixafor derivative for tumor-specific delivery of nanomaterials. Nano Res 11:2159-2172

Le QV, Suh J, Choi JJ, Park GT, Lee JW et al (2019) In situ nanoadjuvant-assembled tumor vaccine for preventing long-term recurrence. ACS Nano 13:7442-7462

Le Q-V, Kim D, Lee J, Shim G, Oh Y-K (2021) Photosensitizer-free phototherapy with peptide micelle nanoadjuvants for cancer vaccine against metastasis of melanoma. Adv Ther 2:2000288

Li Y, He D, Tu J, Wang R, Zu C et al (2018) The comparative effect of wrapping solid gold nanoparticles and hollow gold nanoparticles with doxorubicin-loaded thermosensitive liposomes for cancer thermo-chemotherapy. Nanoscale 10:8628-8641

Liu Y, An X (2019) Preparation, microstructure and function of liposome with light responsive switch. Colloids Surf B Biointerfaces 178:238-244

Liu Y, Zhang X, Luo L, Li L, He Y et al (2018) Self-assembly of stimuli-responsive $\mathrm{Au}-\mathrm{Pd}$ bimetallic nanoflowers based on betulinic acid liposomes for synergistic chemo-photothermal cancer therapy. ACS Biomater Sci Eng 4:2911-2921

Lovell JF, Jin CS, Huynh E, Jin H, Kim C et al (2011) Porphysome nanovesicles generated by porphyrin bilayers for use as multimodal biophotonic contrast agents. Nat Mater 10:324-332

Luo L, Bian Y, Liu Y, Zhang X, Wang M et al (2016) Combined near infrared photothermal therapy and chemotherapy using gold nanoshells coated liposomes to enhance antitumor effect. Small 12:4103-4112

Luo D, Geng J, Li N, Carter KA, Shao S et al (2017) Vessel-targeted chemophototherapy with cationic porphyrin-phospholipid liposomes. Mol Cancer Ther 16:2452-2461

Lyon PC, Griffiths LF, Lee J, Chung D, Carlisle R et al (2017) Clinical trial protocol for TARDOX: a phase I study to investigate the feasibility of targeted release of lyso-thermosensitive liposomal doxorubicin (ThermoDox ${ }^{\circledR}$ ) using focused ultrasound in patients with liver tumours. J Ther Ultrasound 5:28

Mang TS (2004) Lasers and light sources for PDT: past, present and future. Photodiagn Photodyn Ther 1:43-48

Miao W, Shim G, Kang CM, Lee S, Choe YS et al (2013a) Cholesteryl hyaluronic acid-coated, reduced graphene oxide nanosheets for anti-cancer drug delivery. Biomaterials 34:9638-9647

Miao W, Shim G, Lee S, Lee S, Choe YS et al (2013b) Safety and tumor tissue accumulation of pegylated graphene oxide 
nanosheets for co-delivery of anticancer drug and photosensitizer. Biomaterials 34:3402-3410

Moriwaki SI, Misawa J, Yoshinari Y, Yamada I, Takigawa M et al (2001) Analysis of photosensitivity in Japanese cancer-bearing patients receiving photodynamic therapy with porfimer sodium (PhotofrinTM). Photodermatol Photoimmunol Photomed 17:241-243

Pacheco TJA, da Silva VCM, de Souza DG (2020) Nano COVID-19 vaccines: the firsts RNA lipid nanoparticle vaccines being approved from history-review. Res Soc Dev 9:e20191211123-e20191211123

Ribovski L, Zhou Q, Chen J, Feringa BL, van Rijn P et al (2020) Lightinduced molecular rotation triggers on-demand release from liposomes. Chem Commun 56:8774-8777

Salmivuori M, Grönroos M, Tani T, Pölönen I, Räsänen J et al (2020) Hexyl aminolevulinate, 5-aminolevulinic acid nanoemulsion and methyl aminolevulinate in photodynamic therapy of non-aggressive basal cell carcinomas: a non-sponsored, randomized, prospective and double-blinded trial. J Eur Acad Dermatol Venereol 34:2781-2788

Schoenmaker L, Witzigmann D, Kulkarni JA, Verbeke R, Kersten G et al (2021) mRNA-lipid nanoparticle COVID-19 vaccines: structure and stability. Int J Pharm 601:120586

Shah SA, Aslam Khan MUA, Arshad M, Awan SU, Hashmi MU et al (2016) Doxorubicin-loaded photosensitive magnetic liposomes for multi-modal cancer therapy. Colloids Surf B Biointerfaces 148:157-164

Shi J, Su Y, Liu W, Chang J, Zhang Z (2017) A nanoliposomebased photoactivable drug delivery system for enhanced cancer therapy and overcoming treatment resistance. Int $\mathbf{J}$ Nanomed 12:8257-8275

Shim G, Ko S, Kim D, Le QV, Park GT et al (2017) Light-switchable systems for remotely controlled drug delivery. J Control Release 267:67-79

Shim G, Ko S, Park JY, Suh JH, Le QV et al (2020a) Tannic acidfunctionalized boron nitride nanosheets for theranostics. J Control Release 327:616-626

Shim G, Park J, Kim MG, Yang G, Lee Y et al (2020b) Noncovalent tethering of nucleic acid aptamer on DNA nanostructure for targeted photo/chemo/gene therapies. Nanomedicine 24:102053

Swenson CE, Haemmerich D, Maul DH, Knox B, Ehrhart N et al (2015) Increased duration of heating boosts local drug deposition during radiofrequency ablation in combination with thermally sensitive liposomes (ThermoDox) in a porcine model. PLoS ONE 10:e0139752

Tagami T, Ozeki T (2017) Recent trends in clinical trials related to carrier-based drugs. J Pharm Sci 106:2219-2226

Tang X, Wang Z, Zhu Y, Xiao H, Xiao Y (2020) Hypoxia-activated ROS burst liposomes boosted by local mild hyperthermia for photo/chemodynamic therapy. J Control Release 328:100-111

Uda RM, Kato Y, Takei M (2016) Photo-triggered release from liposomes without membrane solubilization, based on binding to poly(vinyl alcohol) carrying a malachite green moiety. Colloids Surf B Biointerfaces 146:716-721

Viitala L, Pajari S, Lajunen T, Kontturi LS, Laaksonen T et al (2016) Photothermally triggered lipid bilayer phase transition and drug release from gold nanorod and indocyanine green encapsulated liposomes. Langmuir 32:4554-4563

Wiraja C, Mathiyazhakan M, Movahedi F, Upputuri PK, Cheng Y (2016) Near-infrared light-sensitive liposomes for enhanced plasmid DNA transfection. Bioeng Transl Med 1:357-364

Wu Y, Li Q, Shim G, Oh YK (2021) Melanin-loaded CpG DNA hydrogel for modulation of tumor immune microenvironment. J Control Release 330:540-553

Yang H, Le QV, Shim G, Oh YK, Shin YK (2020) Molecular engineering of antibodies for site-specific conjugation to lipid polydopamine hybrid nanoparticles. Acta Pharm Sin B 10:2212-2226

Yang Y, Liu X, Ma W, Xu Q, Chen G et al (2021) Light-activatable liposomes for repetitive on-demand drug release and immunopotentiation in hypoxic tumor therapy. Biomaterials 265:120456

Zhang K, Zhang Y, Meng X, Lu H, Chang H et al (2018) Light-triggered theranostic liposomes for tumor diagnosis and combined photodynamic and hypoxia-activated prodrug therapy. Biomaterials 185:301-309

Zhao Y, Zhao T, Cao Y, Sun J, Zhou Q et al (2021) Temperature-sensitive lipid-coated carbon nanotubes for synergistic photothermal therapy and gene therapy. ACS Nano 15:6517-6529

Publisher's Note Springer Nature remains neutral with regard to jurisdictional claims in published maps and institutional affiliations. 